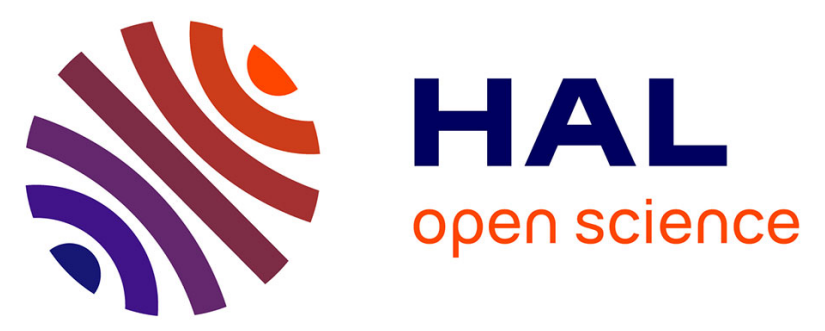

\title{
Refined instrumental variable identification of continuous-time OE and BJ models from irregularly sampled data
}

Fengwei Chen, Hugues Garnier, Marion Gilson

\section{- To cite this version:}

Fengwei Chen, Hugues Garnier, Marion Gilson. Refined instrumental variable identification of continuous-time OE and BJ models from irregularly sampled data. 11th IFAC International Workshop on Adaptation and Learning in Control and Signal Processing, ALCOSP 2013, Jul 2013, Caen, France. pp.CDROM. hal-00906221

\section{HAL Id: hal-00906221 \\ https://hal.science/hal-00906221}

Submitted on 19 Nov 2013

HAL is a multi-disciplinary open access archive for the deposit and dissemination of scientific research documents, whether they are published or not. The documents may come from teaching and research institutions in France or abroad, or from public or private research centers.
L'archive ouverte pluridisciplinaire HAL, est destinée au dépôt et à la diffusion de documents scientifiques de niveau recherche, publiés ou non, émanant des établissements d'enseignement et de recherche français ou étrangers, des laboratoires publics ou privés. 


\title{
Refined Instrumental Variable Identification of Continuous-time OE and BJ Models from Irregularly Sampled Data
}

\author{
F. Chen ${ }^{1,2}$, H. Garnier ${ }^{1,2}$, M. Gilson ${ }^{1,2}$ \\ ${ }^{1}$ University of Lorraine, CRAN, UMR 7039, 2 rue Jean Lamour, \\ F-54519 Vandouvre-les-Nancy, France \\ ${ }^{2}$ CNRS, CRAN, UMR 7039, France \\ fengwei.chen,hugues.garnier,marion.gilson@univ-lorraine.fr
}

\begin{abstract}
This paper looks at the problem of system identification from non-uniformly sampled input-output data. It describes how refined instrumental variable estimators can be derived to directly identify the parameters of continuous-time output error and Box-Jenkins transfer function models from irregularly sampled data. Monte Carlo simulation analysis is used to illustrate the properties of the proposed estimation schemes.
\end{abstract}

Keywords: Continuous-time models, instrumental variable, irregular sampling, parameter estimation, system identification

\section{INTRODUCTION}

Most physical phenomenons are described by continuoustime (CT) models. Despite this fact, the theory of system identification has been developed over many decades mainly based on discrete-time (DT) models estimated from sampled data. Sampling provides the link between CT systems and DT models. When the sampling period is constant and the underlying CT system is linear and time-invariant, the parameters of the DT model can be estimated directly. However, when the sampling is nonuniform (time-variable), the DT model becomes timevarying and in this case, the estimation of DT model parameters becomes more difficult. In this non-uniform sampling situation, it is then more convenient to model the system in CT since the associated parameters remain invariant with respect to the varying sampling period. This is one major advantage of direct CT model identification over DT model identification [H. Garnier and L. Wang (Eds.), 2008]. There are many factors giving rise to non-uniformly sampling, such as manual measurements, faults of machine, losses in data transmission, and other cases where the measurement is not under the control of experimenter so that equidistantly sampling is practically impossible.

CT model identification from non-uniformly sampled data has been recently considered from several different perspectives. For example, in Tsang and Billings [1995], the traditional state-variable filter approach was combined with the orthogonal least-squares to handle the nonuniformly sampled data. More recently, in Goodwin and Cea [2011], a minimum distortion filtering approach is developed while in Larsson et al. [2007], different derivative approximations were considered to identify CT ARX type of models.
This paper looks at two other traditional model structures and focuses more specifically on the use of optimal instrumental variable (IV) methods. One particularly successful implementation of the optimal IV is known as the refined IV method [Young and Jakeman, 1980]. Some recent developments have been recently suggested to handle to SISO and MISO CT transfer function model identification from uniformly sampled data [Garnier et al., 2007, Young et al., 2008]. The aim of this paper is to extend this method to handle non-uniformly sampled data.

First, we develop a refined IV estimator for CT output error (COE) models. As it will be recalled the proposed implementation of the optimal IV solution involves an adaptive procedure of optimal CT prefilters which have to be implemented in a digital way. However, in this nonuniformly sampling situation, special care is required to implement the CT filtering operations. Since only the (irregularly) sampled versions of the CT signals are available, the output of the CT filters can only be computed by using numerical integration methods or discrete-time approximations of these CT filters.

In the second part of the paper, we consider the identification problem of CT Box-Jenkins (BJ) models. This case is not as easy to handle. Indeed, when the sampling is constant, an optimal IV procedure has been recently suggested for identifying hybrid CT BJ models where the model of the dynamic system is estimated in CT form, while the associated additive noise model is estimated as a DT ARMA process [Young et al., 2008]. However in presence of non-uniformly sampled data, this hybrid representation cannot anymore be used easily (the noise model would be time-varying) and therefore full CT models for both plant and noise process is required. This means that estimation methods to CT AR (CAR) or CT ARMA (CARMA) process have to be included in the IVbased estimation schemes. In [Larsson and Söderström, 
2002], the authors provided a method to identify CAR process from irregularly sampled data. Here we restrict the study to the case of a CAR noise model and will use the method suggested in [Larsson and Söderström, 2002]. The resulting model can therefore be considered as a simplified CT BJ model.

This paper is organized in the following way. We first define the parameter estimation problem in Section 2. In Section 3, the optimal IV solution for the general model structure is recalled. Then the optimal IV for OE model identification method including CT filtering implementation aspects is presented in Section 4. The optimal IV solution for identifying CT BJ models based on CAR process estimation is detailed in Section 5. Then, in Section 6, simulation examples are presented to illustrate the properties of the proposed methods.

\section{PROBLEM STATEMENT}

For simplicity of presentation, the formulation and solution of the CT estimation problem will be restricted to the case of a linear, single-input, single-output system. It is assumed that the input $u(t)$ and the noise-free output $x(t)$ are related by the following constant coefficient, differential equation,

$$
\begin{aligned}
& x^{\left(n_{a}\right)}(t)+a_{1}^{o} x^{\left(n_{a}-1\right)}(t)+\cdots+a_{n_{a}}^{o} x(t)= \\
& b_{0}^{o} u^{\left(n_{b}\right)}(t)+\cdots+b_{n_{b}}^{o} u(t)
\end{aligned}
$$

where $x^{(i)}(t)$ denotes the $i$ th time derivative of the continuous-time signal $x(t)$. Equation (1) can also be written in the following compact transfer function (TF) form,

$$
x(t)=G_{o}(p) u(t)=\frac{B_{o}(p)}{A_{o}(p)} u(t)
$$

with

$$
\begin{aligned}
& B_{o}(p)=b_{0}^{o} p^{n_{n}}+b_{1}^{o} p^{m-1}+\cdots+b_{n_{b}}^{o}, \\
& A_{o}(p)=p^{n_{a}}+a_{1}^{o} p^{n_{a}-1}+\cdots+a_{n_{a}}^{o}, \quad n_{a} \geq n_{b}
\end{aligned}
$$

where $p$ is the differential operator, i.e., $p^{i} x(t)=\frac{\mathbf{d}^{i} x(t)}{\mathbf{d} t^{i}}$; $B_{o}(p)$ and $A_{o}(p)$ are assumed to be coprime; and the system is asymptotically stable. It is assumed that the input signal $\left\{u(t), t_{1}<t<t_{N}\right\}$ is applied to the system and this gives rise to an output signal $\left\{x(t), t_{1}<t<t_{N}\right\}$.

It is further assumed that the deterministic output $x(t)$ is corrupted by an additive, coloured measurement noise $\xi(t)$, so that the complete equation for the data-generating system, denoted by $\mathcal{S}$, can be written in the form,

$$
\mathcal{S}: y(t)=G_{o}(p) u(t)+H_{o}(p) e_{o}(t)
$$

or, in the alternative decomposed form that is more appropriate in the present context

$$
\mathcal{S}\left\{\begin{array}{l}
x(t)=G_{o}(p) u(t) \\
\xi(t)=H_{o}(p) e_{o}(t) \\
y(t)=x(t)+\xi(t)
\end{array}\right.
$$

where $H_{o}(p)$ is assumed to be stable and invertible stable, while $e_{o}(t)$ is a zero-mean, stationary continuous-time white noise process of intensity $\sigma_{e_{o}}^{2}$, which is assumed to be uncorrelated with the input $u(t)$. Note that $e_{o}(t)$ is a mathematical abstraction and $y(t)$ results from the sum of a deterministic and a stochastic term.
Of course, in practical situations, the input and output signals $u(t)$ and $y(t)$ will be sampled in discrete time and denoted by $u\left(t_{k}\right)$ and $y\left(t_{k}\right)$. Several papers have discussed the sampling issues of stochastic CT systems (see. e.g. Wahlberg et al. [1993], Ljung and Wills [2010]). For COE models, since $\xi(t)$ is a $\mathrm{CT}$ white noise, it has infinite variance and cannot be measured directly. The output $y(t)$ is thus sampled using the integrated method where a low-pass filter is applied before the sampling. The commonly used prefilter consists of an integrator between sampling times (see Ljung and Wills [2010]). It is furthermore assumed that $u\left(t_{k}\right)$ and $y\left(t_{k}\right)$ are available at irregular time-instants, obtained at a varying sampling interval denoted as

$$
h_{k}=t_{k+1}-t_{k}
$$

It will be also assumed that the sequence of sampling intervals $\left\{h_{k}\right\}$ is a realization of a white stationary random process with an associated probability density function $p_{x}(h)$ and

$$
\underline{h} \leq h_{k} \leq \bar{h}
$$

where $h>0$, and $\bar{h}$ is a finite upper bound. The objective is then to estimate the parameters of the differential equation, based on the non-uniformly sampled input and output data $Z^{N}=\left\{u\left(t_{k}\right) ; y\left(t_{k}\right)\right\}_{k=1}^{N}$.

\section{OPTIMAL IV ESTIMATORS}

In this section we recall the main conditions for obtaining optimal (consistent and minimum variance) IV parameter estimate. Consider the general class of IV estimators

$$
\hat{\boldsymbol{\rho}}=\operatorname{sol}_{\rho} \frac{1}{N} \sum_{k=1}^{N} \boldsymbol{\zeta}_{f}\left(t_{k}\right)\left[y_{f}^{\left(n_{a}\right)}\left(t_{k}\right)-\boldsymbol{\varphi}_{f}^{T}\left(t_{k}\right) \boldsymbol{\rho}\right]=0
$$

where the parameter vector $\boldsymbol{\rho}$ includes the dynamic plant model parameters stacked columnwise as ,

$$
\boldsymbol{\rho}=\left[\begin{array}{llllll}
a_{1} & \cdots & a_{n_{a}} & b_{0} & \cdots & b_{n_{b}}
\end{array}\right]^{T} \in \mathbb{R}^{n_{a}+n_{b}+1}
$$

and $\boldsymbol{\zeta}_{f}\left(t_{k}\right) \in \mathbb{R}^{n_{a}+n_{b}+1}$ is the prefiltered instrumental vector where

$$
\boldsymbol{\zeta}_{f}\left(t_{k}\right)=L(p) \boldsymbol{\zeta}\left(t_{k}\right)
$$

while the output $y\left(t_{k}\right)$ and regression vector $\varphi\left(t_{k}\right)$ are also prefiltered by the same filter $L(p)$ with

$$
\varphi^{T}\left(t_{k}\right)=\left[-y^{\left(n_{a}-1\right)}\left(t_{k}\right) \cdots-y\left(t_{k}\right) u^{\left(n_{b}\right)}\left(t_{k}\right) \cdots u\left(t_{k}\right)\right]
$$

It has been shown that a minimum variance estimator is achieved under the following conditions [Young and Jakeman, 1980] (see also [Söderström and Stoica, 1983]):

$$
\left\{\begin{array}{l}
\zeta_{f}^{\mathrm{opt}}\left(t_{k}\right)=L^{\mathrm{opt}}(p) \stackrel{\circ}{\varphi}\left(t_{k}\right) \\
L^{\mathrm{opt}}(p)=\frac{1}{H_{o}(p) A_{o}(p)}
\end{array}\right.
$$

where $\dot{\varphi}\left(t_{k}\right)$ is the noise-free version of the regression vector $\varphi\left(t_{k}\right)$ defined as

$$
\stackrel{\varphi}{\varphi}\left(t_{k}\right)=\left[-x^{\left(n_{a}-1\right)}\left(t_{k}\right) \cdots-x\left(t_{k}\right) u^{\left(n_{b}\right)}\left(t_{k}\right) \cdots u\left(t_{k}\right)\right]^{T}
$$

The following comments can be made from the IV optimal conditions

(1) Filtering operation is a distinguishing feature of optimal IV solution. This is an interesting feature for the identification problem considered here as the filtering 
will provide a very convenient way of generating the prefiltered derivatives of the input and output variables as required for CT model estimation.

(2) The IV optimality is noise-model dependent and so the way we parametrize the model will influence the implemented solution of the optimal estimates.

(3) The optimal IV estimate is achieved when the instrument variable is equal to the filtered noise-free version of the regression vector.

(4) One particularly successful implementation of the optimal IV is known as the refined IV method where an adaptive procedure blends the separate algorithms for the estimation of the system and noise model parameters using a bootstrap approach. This refined IV method uses an iterative procedure, in which, at each iteration, the auxiliary model is used to generate the instrumental variables and prefilter based on the parameters obtained at the previous iteration. In the next two sections, such refined IV methods are derived to estimate the parameters of CT OE and BJ models from irregularly sampled data.

\section{SRIVC METHOD FOR COE MODELS}

When $H_{o}(p)=1$, the model set to be estimated takes the form of a COE model structure, as denoted by $\mathcal{M}_{\text {coe }}$,

$$
\mathcal{M}_{\text {coe }}\left\{\begin{array}{l}
x(t)=G(p, \boldsymbol{\rho}) u(t) \\
y(t)=x(t)+e_{c}(t)
\end{array}\right.
$$

where $\boldsymbol{\rho}$ is defined in $(8), e_{c}(t)$ is a CT white noise of intensity $\sigma_{e_{c}}^{2}$.

In this COE model setting where the measurement noise is assumed to be white, the refined IV parameter estimates (usually termed as simplified refined IV and abbreviated by SRIVC) are obtained, at iteration $j$, from:

$$
\begin{aligned}
\hat{\boldsymbol{\rho}}^{j}= & {\left[\sum_{k=1}^{N} \boldsymbol{\zeta}_{f}\left(t_{k}, \hat{\boldsymbol{\rho}}^{j-1}\right) \boldsymbol{\varphi}_{f}^{T}\left(t_{k}, \hat{\boldsymbol{\rho}}^{j-1}\right)\right]^{-1} } \\
& {\left[\sum_{k=1}^{N} \boldsymbol{\zeta}_{f}\left(t_{k}, \hat{\boldsymbol{\rho}}^{j-1}\right) y_{f}^{\left(n_{a}\right)}\left(t_{k}, \hat{\boldsymbol{\rho}}^{j-1}\right)\right] }
\end{aligned}
$$

with

$$
\left\{\begin{array}{l}
\boldsymbol{\zeta}_{f}\left(t_{k}, \hat{\boldsymbol{\rho}}^{j-1}\right)=L\left(p, \hat{\boldsymbol{\rho}}^{j-1}\right) \hat{\varphi}\left(t_{k}, \hat{\boldsymbol{\rho}}^{j-1}\right) \\
\boldsymbol{\varphi}_{f}\left(t_{k}, \hat{\boldsymbol{\rho}}^{j-1}\right)=L\left(p, \hat{\boldsymbol{\rho}}^{j-1}\right) \boldsymbol{\varphi}\left(t_{k}\right) \\
y_{f}^{\left(n_{a}\right)}\left(t_{k}, \hat{\boldsymbol{\rho}}^{j-1}\right)=L\left(p, \hat{\boldsymbol{\rho}}^{j-1}\right) y^{\left(n_{a}\right)}\left(t_{k}\right) \\
L\left(p, \hat{\boldsymbol{\rho}}^{j-1}\right)=\frac{1}{A\left(p, \hat{\boldsymbol{\rho}}^{j-1}\right)}
\end{array}\right.
$$

and where $\hat{\varphi}\left(t_{k}, \hat{\rho}^{j-1}\right)$ is an estimate of the noise-free regression vector defined in (12) where the unobserved noise-free output is replaced by an estimate obtained from an auxiliary model based on the parameters estimated at the previous iteration

$$
\hat{x}\left(t_{k}, \hat{\boldsymbol{\rho}}^{j-1}\right)=G\left(p, \hat{\boldsymbol{\rho}}^{j-1}\right) u\left(t_{k}\right)
$$

\subsection{Implementation of the CT filtering}

The SRIVC method for COE models has been proven to be successful in a number of practical cases when the data are regularly sampled [Young and Garnier, 2006]. It is worth noticing that the computation of the SRIVC parameter estimates at iteration $j$ given in (14) requires the value of prefiltered signals at the irregular time-instants $t_{k}$, $k=0, \cdots, N$ in both regression and instrument vectors, expressed below under their developed forms

$$
\begin{aligned}
\boldsymbol{\varphi}_{f}^{T}\left(t_{k}, \hat{\boldsymbol{\rho}}^{j-1}\right)= & {\left[-y_{f}^{\left(n_{a}-1\right)}\left(t_{k}, \hat{\boldsymbol{\rho}}^{j-1}\right) \cdots-y_{f}\left(t_{k}, \hat{\boldsymbol{\rho}}^{j-1}\right)\right.} \\
& \left.u_{f}^{\left(n_{b}\right)}\left(t_{k}, \hat{\boldsymbol{\rho}}^{j-1}\right) \cdots u_{f}\left(t_{k}, \hat{\boldsymbol{\rho}}^{j-1}\right)\right] \\
\boldsymbol{\zeta}_{f}^{T}\left(t_{k}, \hat{\boldsymbol{\rho}}^{j-1}\right)= & {\left[-\hat{x}_{f}^{\left(n_{a}-1\right)}\left(t_{k}, \hat{\boldsymbol{\rho}}^{j-1}\right) \cdots-\hat{x}_{f}\left(t_{k}, \hat{\boldsymbol{\rho}}^{j-1}\right)\right.} \\
& \left.u_{f}^{\left(n_{b}\right)}\left(t_{k}, \hat{\boldsymbol{\rho}}^{j-1}\right) \cdots u_{f}\left(t_{k}, \hat{\boldsymbol{\rho}}^{j-1}\right)\right]
\end{aligned}
$$

This implementation issue is well-known in CT model identification and different methods based on the use of numerical integration methods or DT approximations of the CT filters are usually chosen in the case of regularly sampled data. Here we suggest to employ the fourth-order Runge-Kutta method. This technique is reasonably simple and robust and is a good general candidate for numerical solution of differential equations when combined with an intelligent adaptive step-size routine.

\section{RIVC METHOD FOR CT BJ MODELS}

Consider now the case when the model set to be estimated takes the form of a CT BJ model structure. The structure $\mathcal{S}$ is assumed to have no common factors in the plant $\left(G_{O}\right)$ and noise $\left(H_{o}\right)$ components, so that these models can be parameterised independently. More formally, there exists the following decomposition of the parameter vector $\boldsymbol{\theta}$ for the BJ model,

$$
\boldsymbol{\theta}=\left(\boldsymbol{\rho}^{T} \boldsymbol{\eta}^{T}\right) \in \mathbb{R}^{n_{\rho}+n_{\eta}}
$$

such that the model equations can be written as

$$
\mathcal{M}_{b j}\left\{\begin{array}{l}
x(t)=G(p, \boldsymbol{\rho}) u(t) \\
\xi(t)=H(p, \boldsymbol{\eta}) e_{c}(t) \\
y\left(t_{k}\right)=x\left(t_{k}\right)+\xi\left(t_{k}\right)
\end{array}\right.
$$

where the noise model $H(p, \boldsymbol{\eta})$ takes the more general form of a CARMA model given as

$$
H(p, \boldsymbol{\eta})=\frac{C(p, \boldsymbol{\eta})}{D(p, \boldsymbol{\eta})}=\frac{c_{0} p^{n_{c}}+\cdots+c_{n_{c}-1} p+1}{d_{0} p^{n_{d}}+\cdots+d_{n_{d}-1} p+1}
$$

where it is assumed that $n_{a} \geq n_{d}$. As a CAR model often produces a good approximation of a full CARMA noise model, while being much more simple to estimate, we will consider a CAR model for the noise in the derivation of the optimal IV solution for CT BJ models. A CAR model is given by

$$
H(p, \boldsymbol{\eta})=\frac{1}{D(p, \boldsymbol{\eta})}=\frac{1}{d_{0} p^{n_{d}}+\cdots+d_{n_{d}-1} p+1}
$$

where the associated noise model parameters are stacked columnwise in the parameter vector,

$$
\boldsymbol{\eta}=\left[\begin{array}{lll}
d_{0} & \cdots & d_{n_{d}-1}
\end{array}\right]^{T} \in \mathbb{R}^{n_{d}}
$$

Alternatively, the model (20) can be written in the following vector terms

$$
\mathcal{M}_{b j}\left\{\begin{array}{l}
x^{\left(n_{a}\right)}(t)=\dot{\varphi}^{T}(t) \boldsymbol{\rho} \\
\xi(t)=\phi^{T}(t) \boldsymbol{\eta}+e_{c}(t) \\
y\left(t_{k}\right)=x\left(t_{k}\right)+\xi\left(t_{k}\right)
\end{array}\right.
$$

where $\dot{\varphi}^{T}(t)$ is given in (12) and

$$
\phi^{T}(t)=\left[-\xi^{\left(n_{d}\right)}(t) \cdots-\xi^{(1)}(t)\right]
$$


It must be stressed here that the identification problem is much more complicated in this CT BJ model identification setting. The refined IV bootstrap procedure requires now, at each iteration, the concurrent estimation of the CAR noise model which is discussed in section 5.2.

\subsection{RIVC algorithm}

The complete refined IV (RIVC) algorithm is summarized below.

Step 1. Initialization: Apply the SRIVC algorithm to compute an initial estimate of the plant model parameter vector $\hat{\boldsymbol{\rho}}^{0}$.

\section{Step 2. Iterative IV estimation with prefilters:}

for $j=1$ : convergence

(1) If the estimated plant model is unstable, reflect the unstable zeros of the estimated $\hat{A}\left(p, \hat{\boldsymbol{\rho}}^{j-1}\right)$ polynomial into the stable region of the complex plane ${ }^{1}$. Generate the IV series $\hat{x}\left(t_{k}\right)$ from the system 'auxiliary model':

$$
\hat{x}\left(t_{k}, \hat{\boldsymbol{\rho}}^{j-1}\right)=\frac{\hat{B}\left(p, \hat{\boldsymbol{\rho}}^{j-1}\right)}{\hat{A}\left(p, \hat{\boldsymbol{\rho}}^{j-1}\right)} u\left(t_{k}\right)
$$

with the polynomials based on the estimated parameter vector $\hat{\boldsymbol{\rho}}^{j-1}$ obtained at the previous iteration of the algorithm;

(2) Obtain the latest estimate $\hat{\boldsymbol{\eta}}^{j}$ of the CAR noise model parameters based on the estimated noise sequence

$$
\hat{\xi}\left(t_{k}\right)=y\left(t_{k}\right)-\hat{x}\left(t_{k}, \hat{\boldsymbol{\rho}}^{j-1}\right)
$$

using any consistent CAR estimation algorithm (see section 5.2).

(3) Prefilter the input $u\left(t_{k}\right)$, output $y\left(t_{k}\right)$ and estimated noise-free output $\hat{x}\left(t_{k}, \hat{\boldsymbol{\rho}}^{j-1}\right)$ signals by the filter

$$
L\left(p, \hat{\boldsymbol{\rho}}^{j-1}, \hat{\boldsymbol{\eta}}^{j}\right)=\frac{\hat{D}\left(p, \hat{\boldsymbol{\eta}}^{j}\right)}{\hat{A}\left(p, \hat{\boldsymbol{\rho}}^{j-1}\right)}
$$

with the polynomials based on the estimated parameter vector $\hat{\boldsymbol{\rho}}^{j-1}$ obtained at the previous iteration of the algorithm and $\hat{\boldsymbol{\eta}}^{j}$ obtained in Step (2)

(4) Based on these prefiltered data, compute the estimate $\hat{\boldsymbol{\rho}}^{j}$ of the plant model parameter vector from

$$
\begin{aligned}
\hat{\boldsymbol{\rho}}^{j}= & {\left[\sum_{k=1}^{N} \boldsymbol{\zeta}_{f}\left(t_{k}, \hat{\boldsymbol{\rho}}^{j-1}, \hat{\boldsymbol{\eta}}^{j}\right) \boldsymbol{\varphi}_{f}^{T}\left(t_{k}, \hat{\boldsymbol{\rho}}^{j-1}, \hat{\boldsymbol{\eta}}^{j}\right)\right]^{-1} } \\
& {\left[\sum_{k=1}^{N} \boldsymbol{\zeta}_{f}\left(t_{k}, \hat{\boldsymbol{\rho}}^{j-1}, \hat{\boldsymbol{\eta}}^{j}\right) y_{f}^{\left(n_{a}\right)}\left(t_{k}, \hat{\boldsymbol{\rho}}^{j-1}, \hat{\boldsymbol{\eta}}^{j}\right)\right] }
\end{aligned}
$$

where $\boldsymbol{\zeta}_{f}\left(t_{k}, \hat{\boldsymbol{\rho}}^{j-1}, \hat{\boldsymbol{\eta}}^{j}\right), \boldsymbol{\varphi}_{f}\left(t_{k}, \hat{\boldsymbol{\rho}}^{j-1}, \hat{\boldsymbol{\eta}}^{j}\right)$ are given in (17) and (18) but where a dependency to the CAR end noise model parameter estimates $\hat{\boldsymbol{\eta}}^{j}$ is made clear.

\subsection{Estimation of CAR noise model}

The problem of estimating CAR models from discretetime data, where the data are observed irregularly in time, has been studied for more than two decades (see e.g.

1 This is not essential to the functioning of the algorithm: it allows for rarely occurring situations, normally with very poor data.
[S. Bigi, 1994]). One traditional approach is considered here. Its basic idea is to use linear approximations of the differentiation operator to reconstruct the time-derivatives of the noise from the measured sampled data. Then a linear regression model can be built up and then solved by simple least-squares or IV-based schemes [Larsson and Söderström, 2002]. One solution of this type is briefly recalled and used here. Substituting the $i$ th order differentiation operator $p^{i}$ by a discrete approximation $D^{i}$ leads to

$$
p^{i} f\left(t_{k}\right) \approx D^{i} f\left(t_{k}\right)=\sum_{\mu=0}^{i} \beta_{k}(i, \mu) f\left(t_{k+\mu}\right)
$$

To make approximation (28) concrete, it is natural that $\beta_{k}(i, \mu)$ satisfies the following condition

$$
\sum_{\mu=0}^{i} \beta_{k}(i, \mu)\left(t_{k+\mu}-t_{k}\right)^{\nu}= \begin{cases}i ! & \nu=i \\ 0 & \nu=0, \ldots, i-1\end{cases}
$$

where the solution of $\beta_{k}(i, \mu)$ can be found out as [Larsson and Söderström, 2002]

$$
\beta_{k}(i, \mu)=\frac{i !}{\prod_{\substack{s=0 \\ s \neq \mu}}^{i}\left(t_{k+\mu}-t_{k+s}\right)}, \mu=0, \cdots, i
$$

Consequently, from the second equation in (24), at iteration $j$ of the bootstrap RIVC algorithm, a new linear regression model equation can be reformulated as

$$
\hat{\xi}\left(t_{k}\right)=\hat{\phi}^{T}\left(t_{k}\right) \boldsymbol{\eta}^{j}+\varepsilon\left(t_{k}\right)
$$

where

$$
\hat{\phi}\left(t_{k}\right)=\left[-D^{n_{d}} \hat{\xi}\left(t_{k}\right) \cdots-D^{1} \hat{\xi}\left(t_{k}\right)\right]^{T}
$$

with

$$
\hat{\xi}\left(t_{k}\right)=y\left(t_{k}\right)-\hat{x}\left(t_{k}\right)
$$

where the arguments $\hat{\boldsymbol{\rho}}^{j-1}$ and $\hat{\boldsymbol{\eta}}^{j-1}$ have been dropped from the different variables above to ease of notations.

An IV method is then used to estimate $\boldsymbol{\eta}$. The instrument is built up from a simple delayed version of the noise model output as

$$
\hat{\boldsymbol{\psi}}\left(t_{k}\right)=\left[\begin{array}{lll}
-\hat{\xi}\left(t_{k-1}\right) & \cdots & -\hat{\xi}\left(t_{k-n_{d}-1}\right)
\end{array}\right]^{T}
$$

From $N$ sampled observations, the CAR model parameters can be estimated, at iteration $j$, from

$$
\hat{\boldsymbol{\eta}}^{j}=\left[\sum_{k=1}^{N} \hat{\boldsymbol{\psi}}\left(t_{k}\right) \hat{\boldsymbol{\phi}}^{T}\left(t_{k}, \hat{\boldsymbol{\eta}}^{j-1}\right)\right]^{-1} \sum_{k=1}^{N} \hat{\boldsymbol{\psi}}\left(t_{k}\right) \hat{\xi}\left(t_{k}\right)
$$

\section{NUMERICAL EXAMPLES}

\subsection{Identification of COE models}

Consider the following TF model:

$$
\left\{\begin{array}{l}
x(t)=\frac{5}{p^{2}+2.8 p+4} u(t) \\
y(t)=x(t)+e_{c}(t)
\end{array}\right.
$$

The system has a rise time of about 2 sec. A basic choice for the uniform sampling period recommended for DT model identification would be between $0.2<T_{s}<0.5 \mathrm{sec}$. Twice this upper value will be considered in the following as the upper bound for the irregular sampling period. 
$y(t)$ is sampled using the integrated method presented in [Ljung and Wills, 2010], section 2.

$$
\begin{aligned}
y\left(t_{k}\right) & =\frac{1}{\delta} \int_{t_{k}}^{t_{k}+\delta} y(t) d t=\frac{1}{\delta} \int_{t_{k}}^{t_{k}+\delta} x(t) d t+\frac{1}{\delta} \int_{t_{k}}^{t_{k}+\delta} e_{c}(t) d t \\
& =x_{d}\left(t_{k}\right)+e_{d}\left(t_{k}\right)
\end{aligned}
$$

The input signal $u(t)$ is assumed to be zero-order-hold during the sampling period, here for any $t_{k} \leq t<t_{k+1}$, $u(t)$ satisfies that

$$
u(t)=\sin \left(0.714 t_{k}\right)+\sin \left(1.428 t_{k}\right)+\sin \left(2.142 t_{k}\right)
$$

Moreover, the integral time $\delta$ in (37) is chosen as 0.001 , and $e_{d}\left(t_{k}\right)$ is a DT white noise with variance of $\sigma_{e_{c}}^{2} / \delta$.
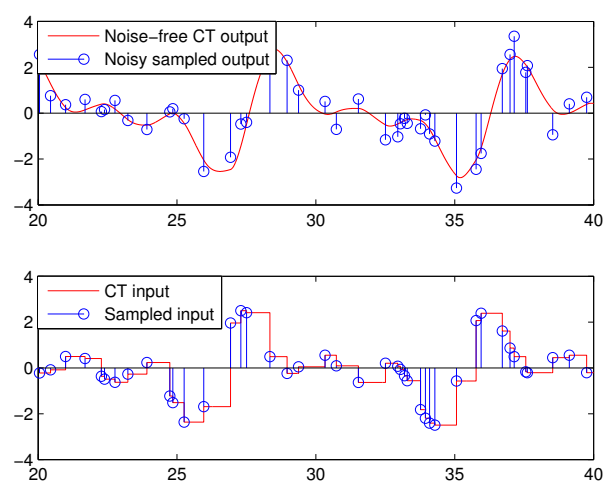

Fig. 1. A portion of the irregularly sampled data set when $\bar{h}=1 s$.

The sampling interval is uniformly distributed over an interval

$$
p_{h_{k}}(x) \sim U(\underline{h}, \bar{h})
$$

The Signal-to-Noise Ratio (SNR) in (37) is set to $10 \mathrm{~dB}$ (here $x_{d}\left(t_{k}\right)$ and $e_{d}\left(t_{k}\right)$ in $(37)$ are termed as signal and noise respectively), this can be realised by adjusting the intensity of $e_{c}(t)$. The number of data is set to $N=1000$. Monte Carlo simulations (MCS) of 200 realizations are performed, with $\underline{h}$ fixed to $0.01 \mathrm{~s}$, while $\bar{h}$ varies from 0.01 to $1 \mathrm{~s}$. When $\underline{h}=\bar{h}=0.01$ s the sampling is uniform. The more $\bar{h}$ increases, the more the sampling deviates from a uniform sampling. Figure 6.1 shows a portion of the observed input-output signals when the upper bound $\bar{h}$ of the sampling period is equal to $1 \mathrm{~s}$.

The iterative algorithm is stopped when

$$
\left\|\frac{\hat{\theta}^{i+1}-\hat{\theta}^{i}}{\hat{\theta}^{i}}\right\|_{\infty} \leq 10^{-5}
$$

where $\|\cdot\|_{\infty}$ is $\infty$-norm operator. This condition is also used in the identification of CT BJ models.

The SRIVC parameter estimates are presented in Figure 2. Furthermore, the results remain very accurate for all $\bar{h}$ over the large considered range where from Figure 2, it may be noticed that the mean and standard deviation of the estimates are almost identical over the range of $\bar{h}$.

Table 6.1 lists the average number of iterations where it can be noted that the SRIVC converges quite quiclky for the different values of $\bar{h}$. The SRIVC algorithm is therefore a very useful and computationally efficient estimator in this non-uniformly samples situation where the additive

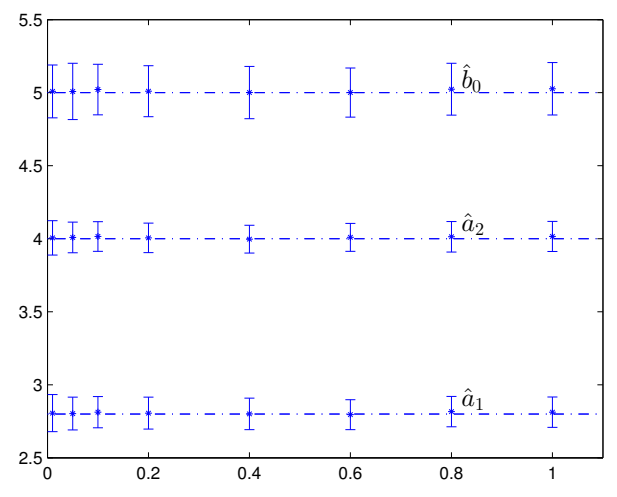

Fig. 2. SRIVC parameter estimates when $h_{k}$ is uniformly distributed. Mean value of the estimates $(*)$. The standard deviation of the estimates are shown by the vertical lines.

\begin{tabular}{|c|c|c|c|c|c|c|c|c|}
\hline $\bar{h}$ & 0.01 & 0.05 & 0.1 & 0.2 & 0.4 & 0.6 & 0.8 & 1 \\
\hline$N_{\text {iter }}$ & 3.7 & 3.8 & 4.0 & 4.2 & 4.6 & 5.4 & 6.4 & 7.4 \\
\hline
\end{tabular}

Table 1. Average number of iterations for convergence

noise is purely white (i.e. $H_{o}(p)=1.0$ ). This algorithm is optimal under these conditions and it functions even if the sampling period varies over a quite large range.

\subsection{Identification of CT BJ models}

Consider now the following continuous-time BJ model given by:

$$
\begin{cases}x(t) & =\frac{5}{p^{2}+2.8 p+4} u(t) \\ \xi(t) & =\frac{1}{0.5 p^{2}+p+1} e_{c}(t) \\ y\left(t_{k}\right) & =x\left(t_{k}\right)+\xi\left(t_{k}\right)\end{cases}
$$

The system is stimulated by the multiline given in (42) and observed at $N=10,000$ time-instants. The sampling pe$\operatorname{riod} h_{k}$ is uniformly distributed over the interval $(0.01, \bar{h})$, where now $\bar{h}$ increases gradually from 0.01 to 0.1 with the step size 0.01 . The noise-free system output is simulated by the Runge-Kutta method.

$$
u(t)=\sin (0.714 t)+\sin (1.428 t)+\sin (2.142 t) .
$$

In order to generate the CAR noise, we have the following derivation. The second equation in (20) can be written into a state-space model

$$
\left\{\begin{array}{l}
\frac{d z(t)}{d t}=A z(t)+B e_{c}(t) \\
\xi(t)=C z(t)
\end{array}\right.
$$

Solving the differential equation, we can obtain a DT statespace model (see e.g. [Wahlberg et al., 1993, Ljung and Wills, 2010])

$$
\left\{\begin{array}{l}
z\left(t_{k}+h_{k}\right)=F_{k} z\left(t_{k}\right)+w\left(t_{k}\right) \\
\xi\left(t_{k}\right)=C z\left(t_{k}\right)
\end{array}\right.
$$

where $F_{k}=e^{A h_{k}}, w\left(t_{k}\right)=\int_{0}^{h_{k}} e^{A t} e_{c}\left(t_{k}+h_{k}-t\right) d t$ $w\left(t_{k}\right)$ is independent DT white and has the following covariance matrix 


$$
\mathbb{E}\left\{w\left(t_{i}\right) w^{T}\left(t_{j}\right)\right\}=\int_{0}^{h_{k}} e^{A t} \sigma_{e_{c}}^{2} e^{A^{T} t} d t \delta_{i, j}
$$

where $\delta_{i, j}$ is Kronecker's delta function.

(44) is used to generate the CAR noise in this paper, which has the same second order properties as the continuoustime one.

Monte Carlo simulations with 100 runs are performed for a SNR set to $10 \mathrm{~dB}$. The RIVC parameter estimates of the plant model are presented in Figure 3, while the noise model parameter estimates are displayed in Figure 4. From these two figures, we can see that both the plant model and the noise model are well estimated.

Another interesting phenomenon is that when $\bar{h}$ increases, the standard deviation reduces slightly. When $\bar{h}$ increases, the time span of the data also increases, that is to say, the sampled data contains more information about the system. This leads to a reduction in parameter estimate variance.

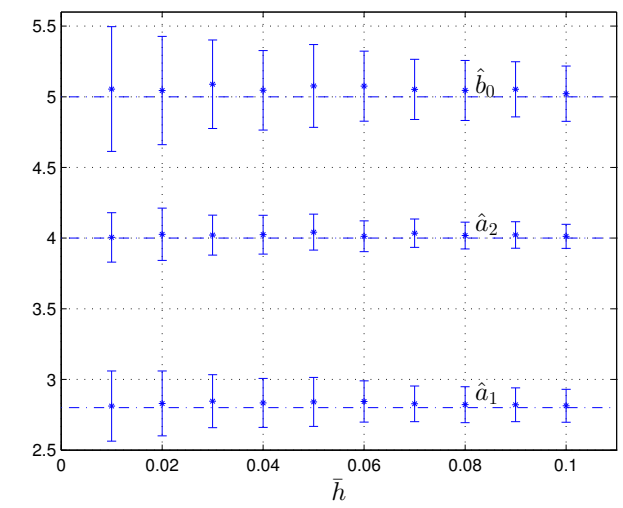

Fig. 3. RIVC Parameter estimates of the plant model when $h_{k}$ is uniformly distributed. The vertical lines stand for deviation of the estimates. $(*)$ stands for the mean estimates.

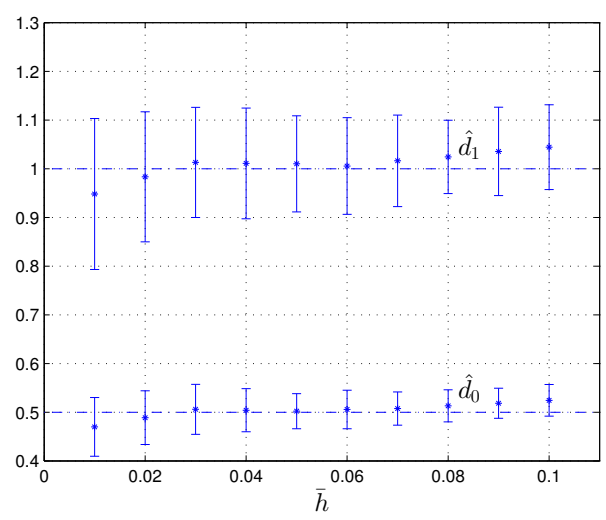

Fig. 4. RIVC parameter estimates of the noise model when $h_{k}$ is uniformly distributed. The vertical lines stand for deviation of the estimates. $(*)$ stands for the mean estimates.

\section{CONCLUSION}

The identification problem of continuous-time output error and Box-Jenkins models from non-uniformly sampled data has been investigated in this paper. Refined instrumental variable-based methods have been developed. Special attention has been paid to the numerical implementation of the continuous-time filtering operations involved in the adaptive procedure. For the BJ model identification, a CAR model identification scheme based on differential operator approximation has been used. The performances of the proposed schemes have been investigated by means of simulation examples. Further research is needed to improve the quality of the BJ model when the sampling time variations are coarse.

\section{REFERENCES}

H. Garnier, M. Gilson, P.C. Young, and E. Huselstein. An optimal IV technique for identifying continuous-time transfer function model of multiple input systems. Control Engineering Practice, 46(15):471-486, April 2007.

G.C. Goodwin and M.G. Cea. Application of minimum distortion filtering to identification of linear systems having non-uniform sampling period. In System Identification, Environmental Modelling, and Control System Design, L. Wang and H. Garnier (Eds.), pages 197-114, Springer-Verlag, 2011. London.

H. Garnier and L. Wang (Eds.), editor. Identification of continuous-time models from sampled data. SpringerVerlag, London, 2008.

E.K. Larsson and T. Söderström. Identification of continuous-time AR processes from unevenly sampled data. Automatica, 38(4):709-718, 2002.

E.K. Larsson, M. Mossberg, and T. Söderström. Identification of continuous-time ARX models from irregularly sampled data. IEEE Transactions on Automatic Control, 52(3):417-427, 2007.

L. Ljung and A. Wills. Issues in sampling and estimating continuous-time models with stochastic disturbances. Automatica, 46:925-931, 2010.

S. Bigi, T. Söderstrström, and C. Carlsson. An IV scheme for estimating continuous-time stochastic models from discrete-time data. In 10th IFAC Symposium on System Identification (SYSID'94), pages 645-650, Copenhagen (Denmark), 1994.

T. Söderström and P. Stoica. Instrumental variable methods for system identification. Springer Verlag, New York, 1983.

K.M. Tsang and S.A. Billings. Identification of systems from non-uniformly sampled data. International Journal of Systems Science, 26(10):1823-1837, 1995.

B. Wahlberg, L. Ljung, and T. Soderstrom. On sampling of continuous-time stochastic processes. Control Theory and Advanced Technology, 9(1):99-112, 1993.

P.C. Young and H. Garnier. Identification and estimation of continuous-time data-based mechanistic (DBM) models for environmental systems. Environmental Modelling and Software, 21(8):1055-1072, August 2006.

P.C. Young and A.J. Jakeman. Refined instrumental variable methods of time-series analysis: Part III, extensions. International Journal of Control, 31:741-764, 1980.

P.C. Young, H. Garnier, and M. Gilson. Refined instrumental variable identification of continuous-time hybrid Box-Jenkins models. In Identification of continuoustime models from sampled data, $\mathrm{H}$. Garnier and L. Wang (Eds.), pages 91-132, Springer-Verlag, 2008, London. 